\title{
Quantitative proteomic analysis of single or fractionated radiation-induced proteins in human breast cancer MDA-MB-231 cells
}

Mi-Hyoung Kim ${ }^{1,2+}$, Seung-Youn Jung ${ }^{1+}$, Jiyeon Ahn ${ }^{1}$, Sang-Gu Hwang ${ }^{1}$, Hee-Jong Woo ${ }^{2}$, Sungkwan An ${ }^{3}$, Seon Young Nam ${ }^{4}$, Dae-Seog Lim ${ }^{5}$ and Jie-Young Song ${ }^{1 *}$

\begin{abstract}
Background: Radiotherapy is widely used to treat cancer alone or in combination with surgery, chemotherapy, and immunotherapy. However, damage to normal tissues and radioresistance of tumor cells are major obstacles to successful radiotherapy. Furthermore, the immune network around tumors appears to be connected to tumor progression and recurrence.

Methods: We investigated the cytosolic proteins produced by irradiated tumor cells by using a quantitative proteomic approach based on stable isotope labeling by amino acids in cell culture. MDA-MB-231 breast cancer cells were treated with a single or fractionated 10 Gray dose of ${ }^{137} \mathrm{Cs}$ y-radiation, which was selected based on cell viability.

Results: Radiation-induced proteins were differentially expressed based on the fractionated times of radiation and were involved in multiple biological functions, including energy metabolism and cytoskeleton organization. We identified 46 proteins increased by at least 1.3-fold, and high ranks were determined for cathepsin D, gelsolin, arginino-succinate synthase 1, peroxiredoxin 5, and C-type mannose receptor 2.
\end{abstract}

Conclusion: These results suggest that a number of tumor-derived factors upregulated by $\gamma$-radiation are promising targets for modulation of the immune response during radiation treatment.

Keywords: Breast cancer, Proteomics, Radiation therapy, SILAC

\section{Background}

Radiation therapy (RT) is one of the most common treatments for cancer and can be provided alone or, frequently, in combination with surgery, chemotherapy, or immunotherapy $[1,2]$. Depending on the type of cancer, $50-90 \%$ of cancer patients undergo RT during the course of their illness, with the highest percentages in cases of non-small-cell lung cancer, prostate, breast, and colon cancer [3,4]. However, RT is associated with acute or chronic side effects such as injury to normal tissues, fatigue, nausea/vomiting, diarrhea, and intestinal bleeding. The effect of radiation on tumor cells varies greatly

\footnotetext{
* Correspondence: immu@kirams.re.kr

${ }^{\dagger}$ Equal contributors

'Division of Radiation Cancer Research, Korea Institute of Radiological \& Medical Sciences, 215-4 Gongneung-dong, Nowon-gu, Seoul 139-706, Korea Full list of author information is available at the end of the article
}

according to the type of radiation, total dose, dose rate, and time of testing post-exposure [4-7]. Therefore, the reasons for RT failure are multiple and vary, and major causes include inadequate vascular supply (hypoxia), cancer stem cells, and novel mutations [8]. Furthermore, tumors can survive in complex systems, including heterogeneous tumor cells, tumor-associated cells, normal cells, and immune cells, rather than as a simple mass of malignant cells. Notably, tumor-associated immune cells produce various signals that are highly predictive of the efficiency of RT as well as cancer progression and recurrence [9].

In the last several years, stimulation of the immune system has been proposed to occur through immunogenic cell death (ICD) mediated mainly by danger signals from endogenous damage-associated molecular patterns (DAMPs) such as stress, damage or injury to tissues, and 
radiation. However, the putative beneficial and detrimental roles of DAMPs in cancer therapy are highly controversial $[7,9]$. Profiling the factors derived by irradiated cancer cells could therefore provide new insights into the prediction of radiation responsiveness.

Breast cancer is the most prevalent malignancy in women and the second leading cause of cancer-related deaths in developed countries. RT is widely used as part of a tri-modal treatment with chemotherapy and surgery; however, approximately $50 \%$ of breast cancer patients have experienced malignant microfoci scattered throughout the breast tissue that can easily progress to metastatic breast cancer [10]. Therefore, new approaches are urgently needed, and multimodal combinatorial therapy is currently being investigated. To this end, we performed a massive quantitative proteomic analysis of irradiated human breast cancer cells (MDA-MB-231) based on stable isotope labeling by amino acids in cell culture (SILAC), which has emerged as one of the most powerful tools for accurate and robust quantitative proteomics, and liquid chromatography-mass spectrometry (LC-MS) [11-13].

In this study, we demonstrated that the tumor-derived proteins commonly increased by both single and fractionated radiation are involved in multiple biological functions. These results indicate promising ICD-associated candidates for cancer treatment that may predict and modify the response to RT.

\section{Methods}

\section{Cell culture}

The human breast cancer cell line MDA-MB-231 was purchased from American Type Culture Collection (ATCC, Manassas, VA, USA). The cells were maintained at $37^{\circ} \mathrm{C}$ in RPMI 1640 with $10 \%$ fetal bovine serum (FBS; Gibco BRL, Grand Island, NY, USA), 100 units $/ \mathrm{mL}$ penicillin, and $100 \mu \mathrm{g} / \mathrm{mL}$ streptomycin in a humidified atmosphere with $5 \% \mathrm{CO}_{2}$.

\section{Cell viability tests}

Cell viability was assessed by trypan blue and MTT (3-[4,5-dimethylthiazol-2-yl]-2,5-diphenyltetrazolium bromide) assays (Sigma-Aldrich, St. Louis, MO, USA) according to the manufacturer's recommendations. MDA-MB-231 cells were plated in 96-well plates $\left(1 \times 10^{4}\right.$ cells/well $)$ in triplicate for indicated times after irradiation. MTT $(0.5$ $\mathrm{mg} / \mathrm{mL}$ ) was added to each well for $3 \mathrm{~h}$, and absorbance was measured at $540 \mathrm{~nm}$ using a microplate reader (Multiskan EX, Thermo LabSystems, Waltham, MA, USA). Additionally, to ascertain cell death, cells were incubated with $2.5 \mathrm{mg} / \mathrm{mL}$ propidium iodide (PI) for 5 min at room temperature and analyzed with a FACSCanto II flow cytometer (Becton Dickson, Franklin Lakes, NJ, USA) and Flowing Software (version 2.5.1; http://www.flowingsoftware.com/).

\section{Stable isotope labeling by amino acids in cell culture} MDA-MB-231 cells were maintained in L-lysine-depleted RPMI 1640 (Invitrogen, Grand Island, NY, USA) supplemented with 10\% dialyzed FBS (Invitrogen, Carlsbad, $\mathrm{CA}, \mathrm{USA}$ ) and $0.1 \mathrm{mg} / \mathrm{mL}$ heavy $\left[\mathrm{U}_{-}{ }^{13} \mathrm{C}_{6}\right]$ or light L-lysine (Invitrogen). Every 3-4 days, cells were split and media replaced with the corresponding light or heavy labeling medium. After approximately six doubling times, cells achieved almost $100 \%$ incorporation of amino acid isotopes. Cells grown with light L-lysine $\left(1 \times 10^{6}\right.$ cells $/ 100$ $\mathrm{mm}$ dish) were exposed to 10 Gray (Gy) ${ }^{137} \mathrm{Cs} \gamma$-radiation (Gammacell 3000 Elan, MDS Nordion, Canada) and harvested after $48 \mathrm{~h}$.

\section{Subcellular fractionation}

An equal ratio (1:1) of treated and untreated MDA-MB231 cells were mixed and fractionated using the ProteoJET ${ }^{\mathrm{Tm}}$ Cytoplasmic and Nuclear Protein Extraction Kit K0311 (Fermentas, Canada) according to the manufacturer's instructions. The efficacy of fractionation was determined via western blotting using GAPDH and Lamin A/C as the cytosolic and nuclear control proteins, respectively. BIOCON (Suwon, Korea) was conducted to identify proteins altered by irradiation.

\section{Database searching}

The tandem mass spectra were extracted, and Sorcerer 3.4 beta2 (Sorcerer software 3.10.4, Sorcerer Web interface 2.2.0.r334) was used to deconvolute and de-isotope the charge states. All of the MS/MS samples were analyzed using Sequest (Thermo Fisher Scientific, San Jose, CA, USA; version 1.3.0.339) set up to search the IPI HUMAN 3.87 database (unknown version, 91464 entries) assuming trypsin digestion. Sequest was searched with a fragment ion mass tolerance of 0.8 or $1.0 \mathrm{Da}$ and a parent ion tolerance of 10.0 PPM. The cysteine iodoacetamide derivative was specified in Sequest as a fixed modification. Deamidated asparagine and glutamine, ${ }^{13} \mathrm{C}$-lysine, ${ }^{13} \mathrm{C}$ - and ${ }^{15} \mathrm{~N}$-arginine, oxidized methionine, acetylated lysine, and phosphorylated serine, threonine, and tyrosine were specified as variable modifications.

\section{Quantitative data analysis}

Scaffold Q+ (version 3.6.2, Proteome Software Inc., Portland, OR, USA) was used to validate MS/MS-based peptide and protein identifications. Peptide identifications were accepted if they were established at greater than $95.0 \%$ probability by the Peptide Prophet algorithm [14]. Protein probabilities were assigned by the Protein Prophet algorithm [15], and identifications were accepted if they were established at greater than $99.0 \%$ probability and contained at least two unique identified peptides. Experimentally acquired intensities were globally normalized across all acquisition runs. Individual quantitative samples 
were normalized within each acquisition run. Intensities for identified peptides were normalized within the assigned protein. The reference channels were normalized to produce a 1:1 fold change. All normalization calculations were performed using medians to multiplicatively normalize data.

\section{Western blot}

Cells were lysed with RIPA buffer (50 mM Tris-Cl, pH 7.4, $1 \% \mathrm{NP}-40,150 \mathrm{mM} \mathrm{NaCl}, 1 \mathrm{mM}$ EDTA) supplemented with protease inhibitors $(1 \mathrm{mM}$ phenylmethylsulfonyl fluoride, $1 \mu \mathrm{g} / \mathrm{mL}$ aprotinin, $1 \mu \mathrm{g} / \mathrm{mL}$ leupeptin, and $1 \mathrm{mM} \mathrm{Na} \mathrm{NO}_{4}$ ). Proteins from whole cell lysates were separated on $8-15 \%$ SDS-polyacrylamide gels and transferred to nitrocellulose membranes (Bio-Rad, Hercules, CA, USA). The membrane was blocked with $5 \%$ skim milk in Tris-buffered saline containing $0.1 \%$ Tween-20 (TBS-T) for $1 \mathrm{~h}$ and probed with primary antibodies overnight at $4^{\circ} \mathrm{C}$. Primary antibodies for cathepsin D (CTSD), gelsolin (GSN), argininosuccinate synthase 1 (ASS1), and C-type mannose receptor 2 (MRC2) were obtained from Santa Cruz Biotechnology (Santa Cruz, CA, USA), and the primary antibody for GAPDH was obtained from AbFrontier (Seoul, Korea). After multiple washes, membranes were incubated with peroxidase-conjugated secondary antibodies, and immunoreactive bands were detected using enhanced chemiluminescence reagents according to the manufacturer's recommendations (GE Healthcare, Little Chalfont, UK). Experiments were repeated at least three times.

\section{Results}

Radiation- induced dose-dependent cytotoxicity in MDA-MB-231 cells

Whereas apoptosis does not activate the immune response, necrotic cell death contributes to inflammation and pathophysiological function through release or secretion of diverse molecules [16]. Therefore, to identify the immune-related molecules produced by tumor cells in response to RT, we first investigated the appropriate radiation dose and harvesting time of MDA-MB-231 cells, highly aggressive and triple-negative breast cancer cells, by trypan blue exclusion (Figure 1A), MTT (Figure 1B), and PI staining (Figure 1C) assays. Exposure to ionizing radiation significantly decreased cell viability in a dose- and time-dependent manner. Cells exposed to radiation above 10 Gy continued to proliferate after $72 \mathrm{~h}$ in the MTT assay but not in the trypan blue exclusion assay, indicating that the methods possess different sensitivities to radiation-induced cell death. In accordance with the trypan blue assay, the percent cell death determined by PI staining increased after irradiation. Cells were harvested $48 \mathrm{~h}$ after $10 \mathrm{~Gy}$ of radiation, chosen to evoke radiation-mediated protein alterations but prevent extensive death, for further experiments. Along with the radiation dose, the effects of fractionated irradiated protocols on normal tissue damage and tumor-host interactions are also important in cancer treatment. Cell survival following single or fractionated irradiation seems to be similar in in vitro studies when the total doses are equal [17]. We also measured cell cytotoxicity after fractionated irradiation up to 10 Gy in 5 daily fractions by trypan blue exclusion (Figure 1D) for comparison with single-dose irradiation. We observed no differences in cell cytotoxicity between single- and fractionated-dose irradiation samples (Additional file 1: Figure S1).

\section{Quantitative proteomic analysis of radiation-induced proteins in MDA-MB-231 cells}

Recently, several studies showed that cells exposed to fractionated radiation exhibit different signatures compared to those treated with a single dose of radiation [18-20]. Therefore, to quantitatively analyze proteome alterations in tumor cells treated with different fractionation regimes of radiation, SILAC-based proteomic analysis was performed. A schematic diagram of the experimental workflow is provided in Figure 2A. MDA-MB-231 cells in light media were treated with $10 \mathrm{~Gy}$ in a single dose (10 Gy $\times 1)$ or in multiple fractionated doses $(2 \mathrm{~Gy} \times 5)$ of radiation. Forty-eight hours after exposure, cytosolic proteins were isolated for analysis (Figure 2B). In duplicated experiments with single dose radiation, 890 and 977 proteins were identified, respectively, with 734 identified in both experiments and 525 quantified. In addition, 847 and 792 proteins were identified after multiple fractionated doses of radiation with 607 identified in both trials and 430 quantified. In total, 314 proteins from MDA-MB-231 cells were simultaneously quantified in both dosing strategies (Figure 2C-E).

\section{Classification of radiation-induced upregulated proteins}

A change of 1.3-2.0-fold is generally used as a cut-off value for significance in SILAC proteomic approaches $[13,21]$. Among the 314 proteins quantified, 46 increased at least 1.3-fold after radiation treatment (Additional file 2: Table S1). For clear comparison of the results, we classified the proteins upregulated at least 1.5 -fold as common to both single and fractionated irradiation, single irradiation-specific-, or fractionated irradiationspecific (Table 1). Ionizing radiation induced the greatest increase in fibronectin 1 (FN1), CTSD, GSN, ASS1, and peroxiredoxin 5 (PRDX5) expression, but the increase in FN1 expression was not statistically significant $(P=0.1451)$.

Although most identified proteins were similarly increased in both single- and fractionated-dose radiation, some proteins such as FN1; chondroitin sulfate 


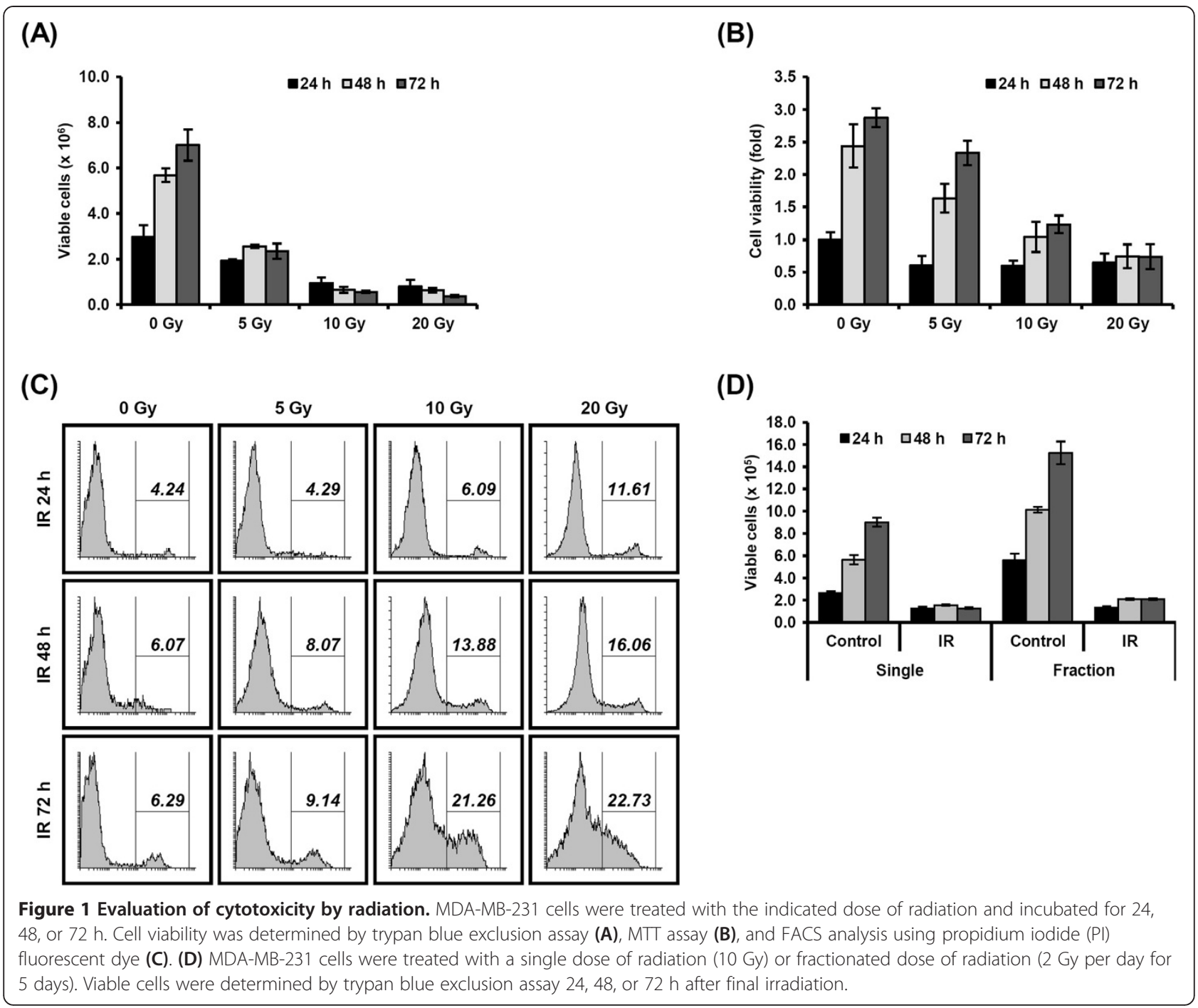

proteoglycan 4 (CSPG4); MRC2; neural precursor cell expressed, developmentally down-regulated 4 (NEDD4); and low-density lipoprotein receptor-related protein 1 (LRP1) were particularly increased by fractionated radiation. In contrast, single-dose radiation increased the expression of proteins, including GSN, AHNAK nucleoprotein, and aldehyde dehydrogenase 2 family, mitochondrial (ALDH2) to a greater extent than fractionated-dose radiation. Additionally, 53 proteins were decreased at least 1.3 -fold by radiation, most of which were identified as ribosomal proteins (Additional file 2: Table S2). Gene ontology (GO) analysis was performed on upregulated proteins using Panther (version 9.0; http://www. pantherdb.org) according to the GO terms for molecular function and biological process [22] (Figure 3A and B). The upregulated proteins were significantly enriched in molecular functions of catalytic activity (33.3\%), binding activity (29.4\%), and structural and molecular activity
(19.6\%). With regard to biological processes, upregulated proteins were involved in metabolic processes $(21.1 \%)$, cellular processes $(21.1 \%)$, localization (15.8\%), cellular component organization or biogenesis (11.6\%), and developmental processes (11.6\%).

\section{Interaction analysis of selected proteins}

Among the top-ranked proteins in SILAC results, five interesting proteins were selected (FN1, CTSD, GSN, ASS1, and MRC2) based on $P$ values and immunological activity potentials. STRING (version 9.1, http://string-db. org) was used to investigate the interaction potential of these proteins [13]. Interaction analysis identified close association of four proteins (FN1, CTSD, GSN, and ASS1; Figure 3C) and indicated potential interactions with cluster of differentiation 44 (CD44), integrin alpha 4 (ITGA4), and integrin alpha 5 (ITGA5), which are involved in cell-cell adhesion and immune response. 


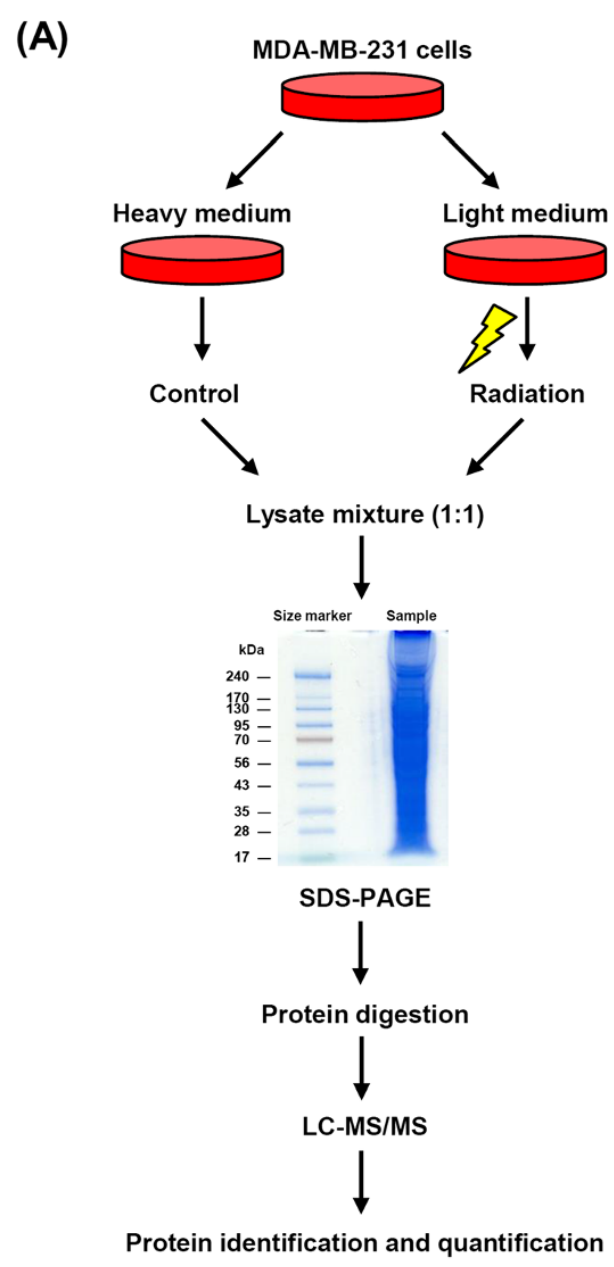

(B)

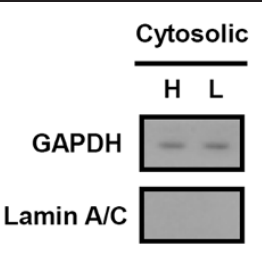

(C)

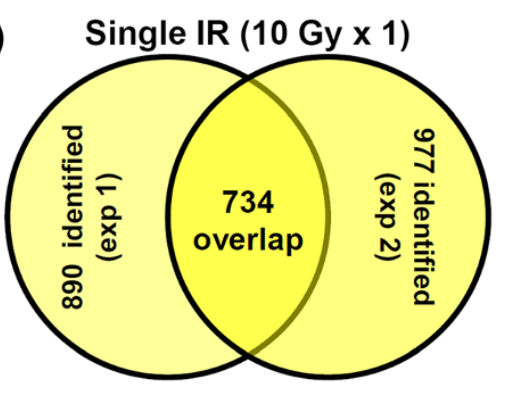

(D) Fractionated IR (2 Gy x 5)

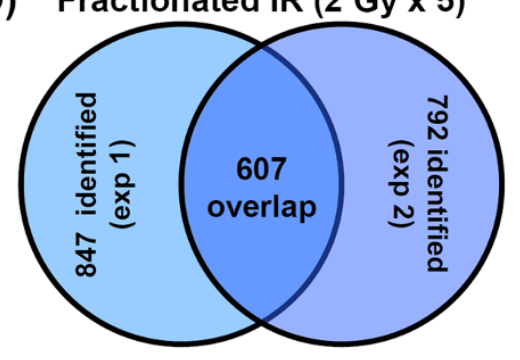

(E)

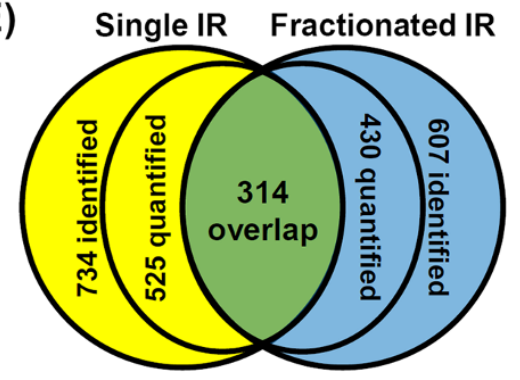

Figure 2 Venn diagram summary of identified proteins by SILAC-based quantitative proteomics. (A) Schematic workflow for profiling radiation-induced proteins via proteomic-based analysis. (B) The cytoplasmic lysates from SILAC-labeled cells were analyzed by western blotting to exclude contamination of nuclear extracts using GAPDH and Lamin A/C as cytosolic and nuclear control proteins, respectively. (C) 734 proteins were identified in the set of single dose experiments, and (D) 607 proteins were identified in the set of fractionated dose experiments.

(E) Comparison of identified proteins from single or fractionated dose of irradiation.

Because MRC2 was not closely linked with the other four, the interaction network of MRC2 was separately verified (Figure 3D), identifying interactions with the plasminogen activator system and anti-inflammatory proteins such as transforming growth factor-beta (TGF- $\beta$ ) and interleukin-10 (IL-10).

\section{Identification of potential proteins selected by} quantitative proteomic analysis

Many previous reports have shown that treatment with ionizing radiation leads to an increase in FN1 expression and poor prognosis [23-25]. Thus, the expression of other candidate proteins after irradiation was investigated to confirm the results of the SILAC proteome approach. The expressions of CTSD and GSN were significantly induced by single-dose radiation in a dose-dependent manner, and the expressions of ASS1 and MRC2 were slightly increased (Figure 4A). Additionally, these four candidates were also significantly upregulated by the fractionated dose of ionizing radiation (Figure 4B). The expression level of candidate proteins induced by a single dose or multiple fractionated doses of irradiation was normalized to GAPDH expression. CTSD is synthesized as an inactive pre-proenzyme that is subsequently converted into an active, intermediate proenzyme $(46 \mathrm{kDa})$ and matures after further cleavage into its more stable two-chain form 
Table 1 Up-regulated proteins were identified by a SILAC-based proteomic approach

\begin{tabular}{|c|c|c|c|c|c|c|c|c|c|}
\hline no & Accession No & Gene symbol & Identified proteins & Fold & $P$ value ${ }^{*}$ & S 1 & S 2 & F 1 & F 2 \\
\hline \multicolumn{10}{|c|}{ Commonly up-regulated proteins } \\
\hline 1 & IPI00022418 & FN1 & Isoform 1 of Fibronectin & 6.525 & 0.0000 & 1.7 & 1.9 & 17.7 & 4.8 \\
\hline 2 & IPI00011229 & CTSD & Cathepsin D & 3.200 & 0.0000 & 2.9 & 3.2 & 3.4 & 3.3 \\
\hline 3 & IPI00026314 & GSN & Isoform 1 of Gelsolin & 2.375 & 0.0000 & 3 & 2.6 & 2 & 1.9 \\
\hline 4 & IPI00020632 & ASS1 & Argininosuccinate synthase & 2.175 & 0.0007 & 2.3 & 2.1 & 2.4 & 1.9 \\
\hline 5 & IPI00024915 & PRDX5 & Isoform Mitochondrial of Peroxiredoxin-5, mitochondrial & 2.025 & 0.0606 & 2.9 & 1.8 & 1.5 & 1.9 \\
\hline 6 & IPI00019157 & CSPG4 & Chondroitin sulfate proteoglycan 4 & 1.900 & 0.3173 & 1.4 & 1 & 2 & 3.2 \\
\hline 7 & IPI00218474 & ENO3 & Isoform 1 of Beta-enolase & 1.900 & 0.0145 & 1 & 1 & 4.6 & 1 \\
\hline 8 & IPI00005707 & MRC2 & C-type mannose receptor 2 & 1.850 & 0.0074 & 1.3 & 1.2 & 2.5 & 2.4 \\
\hline 9 & IPI00940829 & NEDD4 & Isoform 4 of E3 ubiquitin-protein ligase NEDD4 & 1.850 & 0.0004 & 1.3 & 1.3 & 2.4 & 2.4 \\
\hline 10 & IPI00021048 & MYOF & Isoform 1 of Myoferlin & 1.800 & 0.0000 & 1.8 & 1.7 & 2.4 & 1.3 \\
\hline 11 & IPI00021812 & AHNAK & Neuroblast differentiation-associated protein AHNAK & 1.800 & 0.0047 & 1.8 & 2.2 & 1.6 & 1.6 \\
\hline 12 & IPI00020557 & LRP1 & Prolow-density lipoprotein receptor-related protein 1 & 1.800 & 0.0000 & 1.4 & 1.4 & 1.8 & 2.6 \\
\hline 13 & IPI00021766 & RTN4 & Isoform 1 of Reticulon-4 & 1.725 & 0.0019 & 1.6 & 1.5 & 1.7 & 2.1 \\
\hline 14 & IPI00006663 & $\mathrm{ALDH} 2$ & Aldehyde dehydrogenase, mitochondrial & 1.625 & 0.0262 & 2.1 & 1.8 & 1.4 & 1.2 \\
\hline \multicolumn{10}{|c|}{ Single (10 Gy) } \\
\hline 1 & IPI00011229 & CTSD & Cathepsin D & 3.05 & & 2.9 & 3.2 & & \\
\hline 2 & IPI00026314 & GSN & Isoform 1 of Gelsolin & 2.8 & & 3 & 2.6 & & \\
\hline 3 & IPI00024915 & PRDX5 & Isoform Mitochondrial of Peroxiredoxin-5, mitochondrial & 2.35 & & 2.9 & 1.8 & & \\
\hline 4 & IPI00020632 & ASS1 & Argininosuccinate synthase & 2.2 & & 2.3 & 2.1 & & \\
\hline 5 & IPI00021812 & AHNAK & Neuroblast differentiation-associated protein AHNAK & 2 & & 1.8 & 2.2 & & \\
\hline 6 & IPI00006663 & $\mathrm{ALDH} 2$ & Aldehyde dehydrogenase, mitochondrial & 1.95 & & 2.1 & 1.8 & & \\
\hline 7 & IPI00022418 & FN1 & Isoform 1 of Fibronectin & 1.8 & & 1.7 & 1.9 & & \\
\hline 8 & IPI00021048 & MYOF & Isoform 1 of Myoferlin & 1.75 & & 1.8 & 1.7 & & \\
\hline 9 & IPI00021766 & RTN4 & Isoform 1 of Reticulon-4 & 1.55 & & 1.6 & 1.5 & & \\
\hline 10 & IPI00216694 & PLS3 & Plastin-3 & 1.55 & & 1.6 & 1.5 & & \\
\hline 11 & IPI00382844 & $\mathrm{ACO} 2$ & Aconitase (Fragment) & 1.55 & & 1.5 & 1.6 & & \\
\hline 12 & IPI00013860 & $\mathrm{HIBADH}$ & 3-hydroxyisobutyrate dehydrogenase, mitochondrial & 1.55 & & 1.5 & 1.6 & & \\
\hline 13 & IPI00018350 & MCM5 & DNA replication licensing factor MCM5 & 1.55 & & 1.6 & 1.5 & & \\
\hline 14 & IPI00098902 & $\mathrm{OGDH}$ & 2-oxoglutarate dehydrogenase, mitochondrial & 1.5 & & 1.5 & 1.5 & & \\
\hline 15 & IPI00784156 & AP2B1 & Isoform 1 of AP-2 complex subunit beta & 1.5 & & 1.5 & 1.5 & & \\
\hline 16 & IPI00456969 & DYNC1H1 & Cytoplasmic dynein 1 heavy chain 1 & 1.5 & & 1.5 & 1.5 & & \\
\hline \multicolumn{10}{|c|}{ Fraction ( 2 Gy $\times 5$ ) } \\
\hline 1 & IPI00022418 & FN1 & Isoform 1 of Fibronectin & 11.25 & & & & 17.7 & 4.8 \\
\hline 2 & IPI00011229 & CTSD & Cathepsin D & 3.35 & & & & 3.4 & 3.3 \\
\hline 3 & IPI00218474 & ENO3 & Isoform 1 of Beta-enolase & 2.8 & & & & 4.6 & 1 \\
\hline 4 & IPI00019157 & CSPG4 & Chondroitin sulfate proteoglycan 4 & 2.6 & & & & 2 & 3.2 \\
\hline 5 & IPI00005707 & MRC2 & C-type mannose receptor 2 & 2.45 & & & & 2.5 & 2.4 \\
\hline 6 & IPI00940829 & NEDD4 & Isoform 4 of E3 ubiquitin-protein ligase NEDD4 & 2.4 & & & & 2.4 & 2.4 \\
\hline 7 & IPI00020557 & LRP1 & Prolow-density lipoprotein receptor-related protein 1 & 2.2 & & & & 1.8 & 2.6 \\
\hline 8 & IPI00216457 & HIST2H2AA3 & Histone H2A type 2-A & 2.2 & & & & 2.3 & 2.1 \\
\hline 9 & IPI00020632 & ASS1 & Argininosuccinate synthase & 2.15 & & & & 2.4 & 1.9 \\
\hline 10 & IPI00026314 & GSN & Isoform 1 of Gelsolin & 1.95 & & & & 2 & 1.9 \\
\hline 11 & IPI00018398 & PSMC3 & $26 \mathrm{~S}$ protease regulatory subunit $6 \mathrm{~A}$ & 1.95 & & & & 2.8 & 1.1 \\
\hline
\end{tabular}


Table 1 Up-regulated proteins were identified by a SILAC-based proteomic approach (Continued)

\begin{tabular}{|c|c|c|c|c|c|c|}
\hline 12 & IPI00021766 & RTN4 & Isoform 1 of Reticulon-4 & 1.9 & 1.7 & 2.1 \\
\hline 13 & IPI00021048 & MYOF & Isoform 1 of Myoferlin & 1.85 & 2.4 & 1.3 \\
\hline 14 & IPI00293464 & DDB1 & DNA damage-binding protein 1 & 1.75 & 1.4 & 2.1 \\
\hline 15 & IPI00815770 & SNX3 & Isoform 1 of Sorting nexin-3 & 1.75 & 2.3 & 1.2 \\
\hline 16 & IPI00024915 & PRDX5 & Isoform Mitochondrial of Peroxiredoxin-5, mitochondrial & 1.7 & 1.5 & 1.9 \\
\hline 17 & IPI00007682 & ATP6V1A & V-type proton ATPase catalytic subunit A & 1.7 & 1.7 & 1.7 \\
\hline 18 & IPI00021812 & AHNAK & Neuroblast differentiation-associated protein AHNAK & 1.6 & 1.6 & 1.6 \\
\hline 19 & IPI00010418 & MYO1C & Isoform 2 of Myosin-lc & 1.6 & 1.8 & 1.4 \\
\hline 20 & IPI00000005 & NRAS & GTPase NRas & 1.6 & 1.6 & 1.6 \\
\hline 21 & IPI00006482 & ATP1A1 & Isoform Long of Sodium/potassium-transporting ATPase subunit alpha-1 & 1.6 & 1.4 & 1.8 \\
\hline 22 & IPI00023006 & ACTC1 & Actin, alpha cardiac muscle 1 & 1.6 & 2.1 & 1.1 \\
\hline 23 & IPI00221035 & BTF3 & Isoform 1 of Transcription factor BTF3 & 1.6 & 1.6 & 1.6 \\
\hline 24 & IPI00007402 & IPO7 & Importin-7 & 1.55 & 2.2 & 0.9 \\
\hline 25 & IPI00246058 & PDCD6IP & Programmed cell death 6-interacting protein & 1.55 & 1.7 & 1.4 \\
\hline 26 & IPI00026689 & CDK1 & Putative uncharacterized protein DKFZp686L20222 & 1.5 & 1.5 & 1.5 \\
\hline 27 & IPI00001960 & CLIC4 & Chloride intracellular channel protein 4 & 1.5 & 1.4 & 1.6 \\
\hline
\end{tabular}

Proteins increased by an average of 1.5 -fold or more are listed.

*Statistical comparisons were performed using the paired $z$-test, and experimentally verified proteins are represented in bold. S: single-dose irradiation,

F; fractionated-dose irradiation.
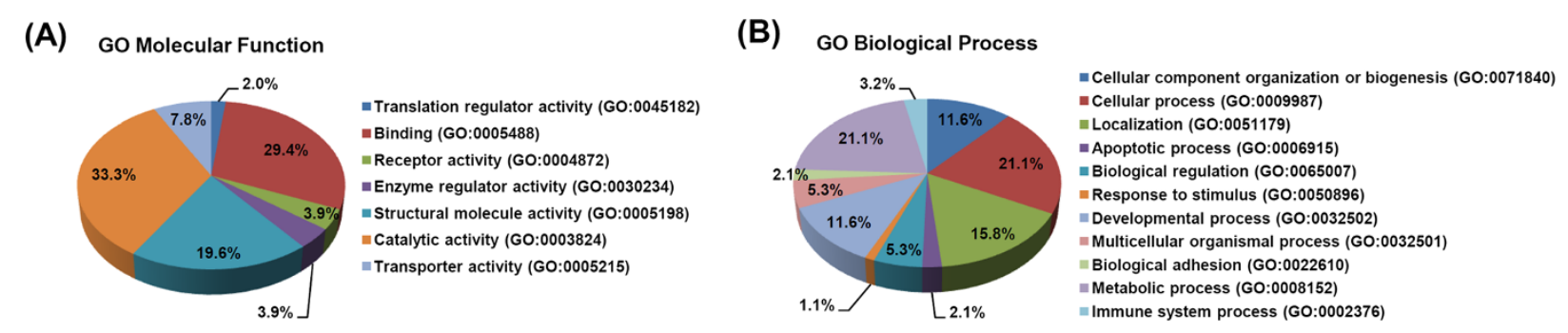

(C)

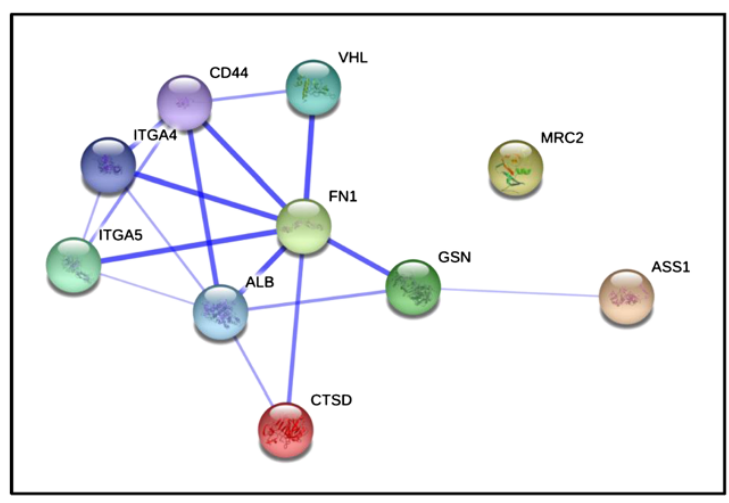

(D)

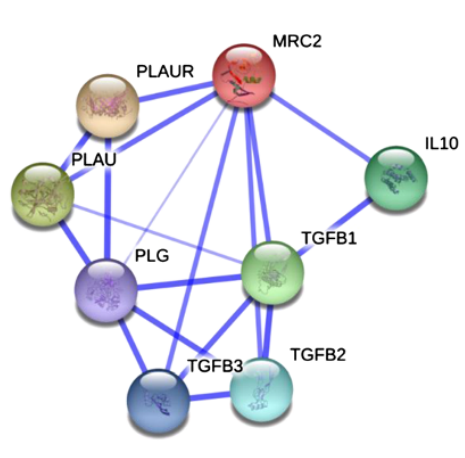

Figure 3 Functional analysis for proteins upregulated after irradiation. Gene ontology (GO) classification was performed according to molecular functions (A) and biological processes (B) using PANTHER (http://www.pantherdb.org/). Interaction network analysis of interesting proteins including FN1, CTSD, GSN, ASS1 (C), and MRC2 (D) was performed using STRING (http://string-db.org). 


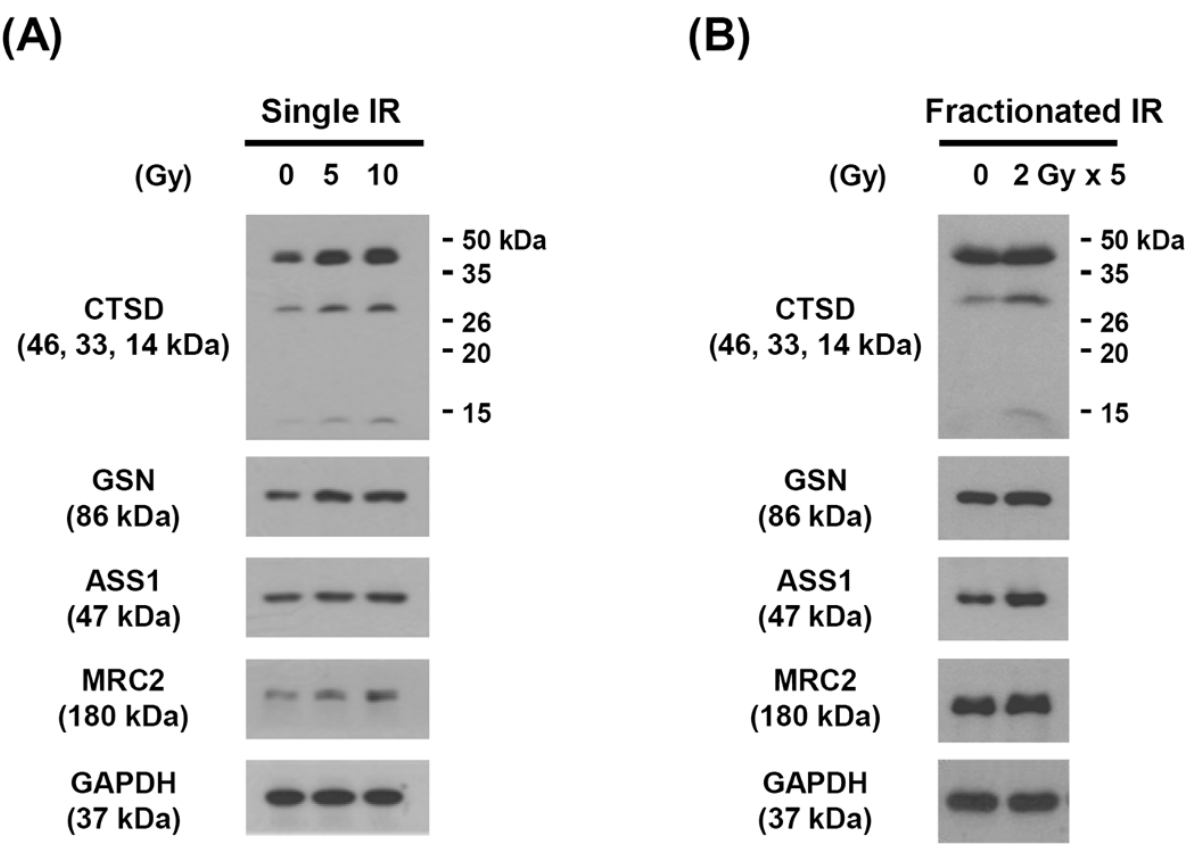

(C)

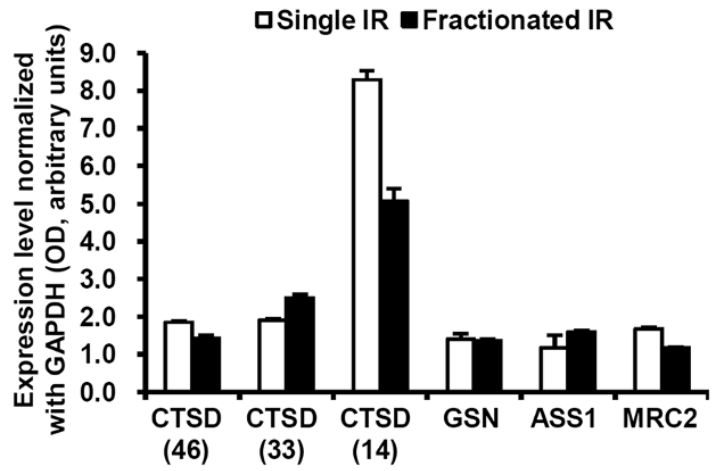

Figure 4 Immunoblot analysis of candidate proteins. MDA-MB-231 cells were exposed to the indicated single (A) or fractionated dose (B) of radiation. After $48 \mathrm{~h}$, total protein was isolated and examined by western blot. (C) Band intensities corresponding to the indicated proteins were quantified by densitometry using ImageJ, normalized to the GAPDH loading control, and expressed as fold-change from each control. Values are mean \pm SD for three independent experiments.

(detected as 33 and $14 \mathrm{kDa}$ ). The bands corresponding to each stage of CTSD cleavage are analyzed in Figure 4C.

\section{Discussion}

Radiation therapy is one of the most effective approaches for cancer treatment $[1,2]$. However, radioresistance and recurrence are major obstacles for the long-term survival of patients undergoing radiation therapy $[2,8]$. Thus, understanding the mechanisms of radiationinduced radioresistance and recurrence is important for the improvement of radiation therapy. In this study, SILAC-based quantitative proteomic analysis was performed to investigate the radiation-induced changes in protein expression in MDA-MB-231 cells with single or fractionated doses of radiation. Although several studies have analyzed protein profiles altered by radiation, this is the first report demonstrating and comparatively analyzing induction of altered molecules by single or fractionated irradiation. Previous studies have employed a single dose of 5-20 Gy radiation, but typical clinical fractionation schedules involve treatment with 1.5-2 Gy per day to a final 60-100 Gy exposure. Moreover, the efficacy of hypofractionated stereotactic body radiotherapy was recently evaluated and attracted attention as a promising curative radiotherapy option. Therefore, it is worthwhile to concurrently determine the proteomic profiles of cells treated with a single or fractionated dose of radiation.

Our results indicate the highest increase in FN1 expression, but the significance of this finding is modest due to different profiles after single or fractionated irradiation. Several studies have shown that ionizing 
radiation-induced FN1 is associated with radiation resistance, cell motility, and fibrosis [26-30]. Since FN1 expression may be greatly affected by fractionated radiation therapy, fractionated irradiation may cause more resistance than single dose irradiation. In various human cancers, especially breast cancer, high levels of CTSD expression were also reported [31]. Generally, CTSD plays a role in apoptosis, innate immune response, and inflammation but also stimulates growth, invasion, migration, angiogenesis [32,33], and cellular senescence in cancer cells $[34,35]$. Thus, the function of increased CTSD expression in cancer cells requires further investigation.

The interaction network of radiation-induced proteins was examined through STRING analysis. Interestingly, interactions between FN1, CTSD, GSN, and ASS1 were indicated, and these proteins were linked with ITGA4, ITGA5, and CD44. ITGA4 and ITGA5 are well-known fibronectin receptors that are altered in many cancer types [27,36]. ITGA4 is overexpressed in chronic lymphocytic leukemia and associated with migration and retention in lymph node and bone marrow tissues. ITGA4 also promotes colony formation, drug resistance, and tumor-initiation in breast sarcomas [37,38]. Similar to ITGA4, ITGA5 is involved in cell proliferation, survival, apoptosis, and angiogenesis [21,27]. In addition, CD44 is responsible for cell adhesion, cell-cell interaction, lymphocyte activation, and tumor metastasis $[39,40]$. GSN is a multifunctional actin-binding protein that controls the length of actin filaments through severing, capping, and nucleating activities. Changes in GSN level are observed in numerous human tumors and are closely related to higher migration capacity, development, and progression of cancer [41]. Therefore, we hypothesize that the FN1, CTSD, and GSN interaction is associated with cell adhesion and metastasis in tumor cells.

Several reports have suggested that MRC2 plays a role in extracellular matrix remodeling and metastasis $[42,43]$. Through the STRING analysis, we found that MRC2 is linked with plasminogen activator system, TGF- $\beta$, and IL-10. In tumor tissues, plasminogen activator system is closely related to angiogenesis, fibrosis, and metastasis [44-46]. Moreover, TGF- $\beta$ is also a wellknown factor in immunosuppression, fibrosis, and the epithelial-mesenchymal transition (EMT) in tumor cells [47-49]. The function of anti-inflammatory cytokine IL-10 in tumor cells remains controversial as it has both tumor-suppressive and oncogenic roles [50,51].

Several studies have demonstrated that migration and invasion are promoted by irradiation in breast cancer, pancreatic cancer, glioma, melanoma, rectal carcinoma, and colon carcinoma cells [52-58]. Our results suggest that radiation-induced increases of FN1, CTSD, GSN, and MRC2 may play radioresistant and negative roles in cancer therapy. Tumor cells killed by radiation treatment may release molecules unexpectedly but concomitantly produce favorable factors that protect undamaged tumor cells, thus increasing invasion or metastasis. However, the direct effect of those molecules on immune cells has not been investigated, so candidate proteins can possibly act as danger signals to stimulate the immune response for eradication of tumor cells after irradiation [16]. Further study is required to reveal the function of radiationmediated protein expression in dendritic cells and provide good candidates for increasing radiosensitivity.

\section{Conclusion}

Our results demonstrate that CTSD, GSN, and MRC2 are significantly increased in irradiated MDA-MB-231 cells and may be promising targets for predicting or enhancing the results of radiation therapy. Interest in combining radiotherapy with other immunotherapeutic agents, such as tumor vaccines, adoptive transfer of immune cells, and treatment with immune checkpoint blockers, is increasing. This study provides a front line approach for establishing the best condition for combined cancer therapeutics.

\section{Additional files}

\section{Additional file 1: Figure S1. Evaluation of cytotoxicity by single or fractionated radiation. MDA-MB-231 cells were treated with single (10 Gy) or fractionated (2 Gy per day for 5 days) dose of radiation. Cell viability was determined by the MTT assay (A) or FACS analysis using propidium iodide (PI) fluorescent dye (B) 24, 48, or 72 h after the last irradiation. \\ Additional file 2: Table S1. Up-regulated proteins were identified by a SILAC-based proteomic approach. Table S2. Down-regulated proteins were identified by a SILAC-based proteomic approach.}

\section{Abbreviations}

ALDH2: Aldehyde dehydrogenase 2 family, mitochondrial; ASS1: Argininosuccinate synthase 1; CD44: Cluster of differentiation 44; CSPG4: Chondroitin sulfate proteoglycan 4; CTSD: Cathepsin D; DAMP: Damage-associated molecular patterns; EMT: Epithelial-mesenchymal transition; FBS: Fetal bovine serum; FN1: Fibronectin 1; GO: Gene ontology; GSN: Gelsolin; Gy: Gray; ICD: Immunogenic cell death; IL-10: Interleukin-10; ITGA4: Integrin alpha 4; ITGA5: Integrin alpha 5; LC-MS: Liquid chromatography-mass spectrometry; LRP1: Low-density lipoprotein receptor-related protein 1; MRC2: C-type mannose receptor 2; MTT: 3-[4,5-dimethylthiazol-2-yl]-2,5-diphenyltetrazolium bromide; NEDD4: Neural precursor cell expressed, developmentally down-regulated 4; PI: Propidium iodide; PRDX5: Peroxiredoxin 5; RT: Radiation therapy; SILAC: Stable isotope labeling by amino acids in cell culture; TBS-T: Tris-buffered saline containing 0.1\% Tween-20; TGF- $\beta$ : Transforming growth factor-beta.

\section{Competing interests}

The authors declare they have no competing interest.

\section{Authors' contributions}

MHK and SYJ carried out the proteomic analysis and immunoassays and drafted the manuscript. JA, HJW, and DSL conceived of and participated in the design of the study. SGH, S An, and SYN analyzed and interpreted the data and helped revise the manuscript. JYS conceived of the study, participated in the design of the study, and helped revise the manuscript. All authors read and approved the final manuscript. 


\section{Acknowledgements}

This work was supported by the Korean Health Technology R\&D Project (Grant HI11C1798) of the Ministry of Health \& Welfare and by Grant No. NRF-2013M2A2A7028423 from the Nuclear Research \& Development Program of the National Research Foundation of Korea.

\section{Author details}

${ }^{1}$ Division of Radiation Cancer Research, Korea Institute of Radiological \& Medical Sciences, 215-4 Gongneung-dong, Nowon-gu, Seoul 139-706, Korea. ${ }^{2}$ Laboratory of Immunology, College of Veterinary Medicine, Seoul National University, Seoul, Korea. ${ }^{3}$ Department of Microbiological Engineering, Kon-Kuk University, Seoul, Korea. ${ }^{4}$ Radiation Health Institute, Korea Hydro \& Nuclear Power Co., Ltd, Seoul, Korea. ${ }^{5}$ Department of Applied Bioscience, CHA University, Gyeonggi-do, Korea.

Received: 25 August 2014 Accepted: 2 December 2014 Published: 3 February 2015

\section{References}

1. Ahmad SS, Duke S, Jena R, Williams MV, Burnet NG. Advances in radiotherapy. BMJ. 2012;345:e7765.

2. Begg AC, Stewart FA, Vens C. Strategies to improve radiotherapy with targeted drugs. Nat Rev Cancer. 2011;11:239-53.

3. Delaney $\mathrm{G}$, Jacob S, Featherstone C, Barton M. The role of radiotherapy in cancer treatment: estimating optimal utilization from a review of evidence-based clinical guidelines. Cancer. 2005;104:1129-37.

4. Baskar R, Lee KA, Yeo R, Yeoh K-W. Cancer and radiation therapy: current advances and future directions. Int J Med Sci. 2012;9:193-9.

5. Berrington De Gonzalez A, Gilbert E, Curtis R, Inskip P, Kleinerman R, Morton $L$, et al. Second solid cancers after radiation therapy: a systematic review of the epidemiologic studies of the radiation dose-response relationship. Int J Radiat Oncol Biol Phys. 2013;86:224-33.

6. Jereczek-Fossa BA, Marsiglia HR, Orecchia R. Radiotherapy-related fatigue. Crit Rev Oncol Hematol. 2002;41:317-25.

7. Vacchelli E, Vitale I, Tartour E, Eggermont A, Sautès-Fridman C, Galon J, et al. Trial watch: anticancer radioimmunotherapy. Oncoimmunol. 2013;2:e25595.

8. Ogawa K, Yoshioka Y, Isohashi F, Seo Y, Yoshida K, Yamazaki H. Radiotherapy targeting cancer stem cells: current views and future perspectives. Anticancer Res. 2013;33:747-54.

9. Levy A, Chargari C, Cheminant M, Simon N, Bourgier C, Deutsch E. Radiation therapy and immunotherapy: implications for a combined cancer treatment. Crit Rev Oncol Hematol. 2013;85:278-87.

10. Holland R, Veling SH, Mravunac M, Hendriks JH. Histologic multifocality of Tis, T1-2 breast carcinomas. Implications for clinical trials of breastconserving surgery. Cancer. 1985;56:979-90.

11. Mann M. Functional and quantitative proteomics using SILAC. Nat Rev Mol Cell Biol. 2006;7:952-8.

12. Stenberg J, Rüetschi U, Skiöldebrand E, Kärrholm J, Lindahl A. Quantitative proteomics reveals regulatory differences in the chondrocyte secretome from human medial and lateral femoral condyles in osteoarthritic patients. Proteome Sci. 2013;11:43.

13. Zeng $X$, Yang $P$, Chen $B$, Jin $X$, Liu $Y$, Zhao $X$, et al. Quantitative secretome analysis reveals the interactions between epithelia and tumor cells by in vitro modulating colon cancer microenvironment. J Proteomics. 2013;89:51-70

14. Keller A, Nesvizhskii Al, Kolker E, Aebersold R. Empirical statistical model to estimate the accuracy of peptide identifications made by MS/MS and database search. Anal Chem. 2002;74:5383-92.

15. Nesvizhskii Al, Keller A, Kolker E, Aebersold R. A statistical model for identifying proteins by tandem mass spectrometry. Anal Chem. 2003;75:4646-58

16. Basu S, Binder RJ, Suto R, Anderson KM, Srivastava PK. Necrotic but not apoptotic cell death releases heat shock proteins, which deliver a partial maturation signal to dendritic cells and activate the NF-kappa B pathway. Int Immunol. 2000;12:1539-46.

17. Verbakel WFAR, van den Berg J, Slotman BJ, Sminia P. Comparable cell survival between high dose rate flattening filter free and conventional dose rate irradiation. Acta Oncol Stockh Swed. 2013;52:652-7.

18. John-Aryankalayil M, Palayoor ST, Cerna D, Simone CB, Falduto MT, Magnuson SR, et al. Fractionated radiation therapy can induce a molecular profile for therapeutic targeting. Radiat Res. 2010;174:446-58.
19. John-Aryankalayil M, Palayoor ST, Makinde AY, Cerna D, Simone CB, Falduto MT, et al. Fractionated radiation alters oncomir and tumor suppressor miRNAs in human prostate cancer cells. Radiat Res. 2012;178:105-17.

20. Schaue D, Ratikan JA, Iwamoto KS, McBride WH. Maximizing tumor immunity with fractionated radiation. Int J Radiat Oncol Biol Phys. 2012;83:1306-10.

21. Fang Z, Yao W, Xiong Y, Zhang J, Liu L, Li J, et al. Functional elucidation and methylation-mediated downregulation of ITGA5 gene in breast cancer cell line MDA-MB-468. J Cell Biochem. 2010;110:1130-41.

22. Zhang H, Xu Y, Filipovic A, Lit LC, Koo C-Y, Stebbing J, et al. SILAC-based phosphoproteomics reveals an inhibitory role of KSR1 in p53 transcriptional activity via modulation of DBC1. Br J Cancer. 2013;109:2675-84.

23. Bae YK, Kim A, Kim MK, Choi JE, Kang SH, Lee SJ. Fibronectin expression in carcinoma cells correlates with tumor aggressiveness and poor clinical outcome in patients with invasive breast cancer. Hum Pathol. 2013;44:2028-37.

24. Jerhammar F, Ceder R, Garvin S, Grénman R, Grafström RC, Roberg K. Fibronectin 1 is a potential biomarker for radioresistance in head and neck squamous cell carcinoma. Cancer Biol Ther. 2010;10:1244-51.

25. Park S, Ahn J-Y, Lim M-J, Kim M-H, Yun Y-S, Jeong G, et al. Sustained expression of NADPH oxidase 4 by p38 MAPK-Akt signaling potentiates radiation-induced differentiation of lung fibroblasts. J Mol Med Berl Ger. 2010;88:807-16.

26. Cordes N, Blaese MA, Plasswilm L, Rodemann HP, Van Beuningen D. Fibronectin and laminin increase resistance to ionizing radiation and the cytotoxic drug Ukrain in human tumour and normal cells in vitro. Int J Radiat Biol. 2003;79:709-20.

27. Crisa L, Cirulli V, Ellisman MH, Ishii JK, Elices MJ, Salomon DR. Cell adhesion and migration are regulated at distinct stages of thymic T cell development: the roles of fibronectin, VLA4, and VLA5. J Exp Med. 1996;184:215-28.

28. Li X, Qian H, Ono F, Tsuchisaka A, Krol RP, Ohara K, et al. Human dermal fibroblast migration induced by fibronectin in autocrine and paracrine manners. Exp Dermatol. 2014;23:682-4.

29. Yokoi H, Mukoyama M, Sugawara A, Mori K, Nagae T, Makino H, et al. Role of connective tissue growth factor in fibronectin expression and tubulointerstitial fibrosis. Am J Physiol Renal Physiol. 2002;282:F933-42.

30. Yousif NG. Fibronectin promotes migration and invasion of ovarian cancer cells through up-regulation of FAK-PI3K/Akt pathway. Cell Biol Int. 2014;38:85-91.

31. Wolf M, Clark-Lewis I, Buri C, Langen H, Lis M, Mazzucchelli L. Cathepsin D specifically cleaves the chemokines macrophage inflammatory protein-1 alpha, macrophage inflammatory protein-1 beta, and SLC that are expressed in human breast cancer. Am J Pathol. 2003;162:1183-90.

32. Benes P, Vetvicka V, Fusek M. Cathepsin D-many functions of one aspartic protease. Crit Rev Oncol Hematol. 2008;68:12-28.

33. Masson O, Bach A-S, Derocq D, Prébois C, Laurent-Matha $V$, Pattingre $S$, et al. Pathophysiological functions of cathepsin D: Targeting its catalytic activity versus its protein binding activity? Biochimie. 2010;92:1635-43.

34. Byun H-O, Han N-K, Lee H-J, Kim K-B, Ko Y-G, Yoon G, et al. Cathepsin D and eukaryotic translation elongation factor 1 as promising markers of cellular senescence. Cancer Res. 2009;69:4638-47.

35. Kim Y-J, Go H, Wu H-G, Jeon YK, Park SW, Lee SH. Immunohistochemical study identifying prognostic biomolecular markers in nasopharyngeal carcinoma treated by radiotherapy. Head Neck. 2011;33:1458-66.

36. Desgrosellier JS, Cheresh DA. Integrins in cancer: biological implications and therapeutic opportunities. Nat Rev Cancer. 2010;10:9-22.

37. Lee K-M, Han W, Kim JB, Shin I, Ko E, Park IA, et al. The CD49d+/high subpopulation from isolated human breast sarcoma spheres possesses tumor-initiating ability. Int J Oncol. 2012;40:665-72.

38. Zucchetto A, Caldana C, Benedetti D, Tissino E, Rossi FM, Hutterer E, et al. CD49d is overexpressed by trisomy 12 chronic lymphocytic leukemia cells: evidence for a methylation-dependent regulation mechanism. Blood. 2013;122:3317-21.

39. Marhaba R, Zöller M. CD44 in cancer progression: adhesion, migration and growth regulation. J Mol Histol. 2004;35:211-31.

40. Sneath RJ, Mangham DC. The normal structure and function of CD44 and its role in neoplasia. Mol Pathol MP. 1998;51:191-200.

41. Litwin M, Nowak D, Mazur AJ, Baczyńska D, Mannherz HG, Malicka-Błaszkiewicz M. Gelsolin affects the migratory ability of human colon adenocarcinoma and melanoma cells. Life Sci. 2012;90:851-61.

42. Engelholm LH, Ingvarsen S, Jürgensen HJ, Hillig T, Madsen DH, Nielsen BS, et al. The collagen receptor UPARAP/Endo180. Front Biosci Landmark Ed. 2009;14:2103-14. 
43. Palmieri C, Caley MP, Purshouse K, Fonseca A-V, Rodriguez-Teja M, Kogianni $\mathrm{G}$, et al. Endo180 modulation by bisphosphonates and diagnostic accuracy in metastatic breast cancer. Br J Cancer. 2013;108:163-9.

44. Binder BR, Mihaly J, Prager GW. UPAR-UPA-PAI-1 interactions and signaling: a vascular biologist's view. Thromb Haemost. 2007;97:336-42.

45. Booth NA. Fibrinolysis and thrombosis. Baillières Best Pract Res Clin Haematol. 1999;12:423-33.

46. Mazar AP, Henkin J, Goldfarb RH. The urokinase plasminogen activator system in cancer: implications for tumor angiogenesis and metastasis. Angiogenesis. 1999;3:15-32.

47. Moustakas A, Heldin P. TGF $\beta$ and matrix-regulated epithelial to mesenchymal transition. Biochim Biophys Acta. 2014;1840:2621-34.

48. Oh SA, Li MO. TGF- $\beta$ : guardian of T cell function. J Immunol Baltim Md 1950. 2013;191:3973-9.

49. Principe DR, Doll JA, Bauer J, Jung B, Munshi HG, Bartholin L, et al. TGF- $B$ : duality of function between tumor prevention and carcinogenesis. J Natl Cancer Inst. 2014;106:djt369.

50. Mocellin S, Panelli MC, Wang E, Nagorsen D, Marincola FM. The dual role of IL-10. Trends Immunol. 2003;24:36-43.

51. Wang Y, Liu X-H, Li Y-H, Li O. The paradox of IL-10-mediated modulation in cervical cancer. Biomed Rep. 2013;1:347-51.

52. De Bacco F, Luraghi P, Medico E, Reato G, Girolami F, Perera T, et al. Induction of MET by ionizing radiation and its role in radioresistance and invasive growth of cancer. J Natl Cancer Inst. 2011;103:645-61.

53. Ohuchida K, Mizumoto K, Murakami M, Qian L-W, Sato N, Nagai E, et al. Radiation to stromal fibroblasts increases invasiveness of pancreatic cancer cells through tumor-stromal interactions. Cancer Res. 2004;64:3215-22.

54. Park C-M, Park M-J, Kwak H-J, Lee H-C, Kim M-S, Lee S-H, et al. lonizing radiation enhances matrix metalloproteinase-2 secretion and invasion of glioma cells through Src/epidermal growth factor receptor-mediated p38/Akt and phosphatidylinositol 3-kinase/Akt signaling pathways. Cancer Res. 2006;66:8511-9.

55. Rofstad EK, Mathiesen B, Galappathi K. Increased metastatic dissemination in human melanoma xenografts after subcurative radiation treatment: radiation-induced increase in fraction of hypoxic cells and hypoxia-induced up-regulation of urokinase-type plasminogen activator receptor. Cancer Res. 2004;64:13-8.

56. Kaliski A, Maggiorella L, Cengel KA, Mathe D, Rouffiac V, Opolon P, et al. Angiogenesis and tumor growth inhibition by a matrix metalloproteinase inhibitor targeting radiation-induced invasion. Mol Cancer Ther. 2005:4:1717-28.

57. Speake WJ, Dean RA, Kumar A, Morris TM, Scholefield JH, Watson SA Radiation induced MMP expression from rectal cancer is short lived but contributes to in vitro invasion. Eur J Surg Oncol J Eur Soc Surg Oncol Br Assoc Surg Oncol. 2005;31:869-74.

58. Wang JL, Sun Y, Wu S. Gamma-irradiation induces matrix metalloproteinase II expression in a p53-dependent manner. Mol Carcinog. 2000;27:252-8.

doi:10.1186/2045-3701-5-2

Cite this article as: Kim et al:: Quantitative proteomic analysis of single or fractionated radiation-induced proteins in

human breast cancer MDA-MB-231 cells. Cell \& Bioscience 2015 5:2.

\section{Submit your next manuscript to BioMed Central and take full advantage of:}

- Convenient online submission

- Thorough peer review

- No space constraints or color figure charges

- Immediate publication on acceptance

- Inclusion in PubMed, CAS, Scopus and Google Scholar

- Research which is freely available for redistribution

Submit your manuscript at www.biomedcentral.com/submit 